\title{
5 Research Square

\section{Prognostic Factors in Resected Pancreatic Ductal Adenocarcinoma: Is Neutrophil-Lymphocyte Ratio a Useful Marker?}

Ignacio Guillermo Merlo ( $\sim$ ignacio.merlo@hospitalitaliano.org.ar)

Hospital Italiano de Buenos Aires https://orcid.org/0000-0002-5913-1836

\section{Victoria Ardiles}

Hospital Italiano de Buenos Aires

Rodrigo Sanchez-Clariá

Hospital Italiano de Buenos Aires

\section{Eugenia Fratantoni}

Hospital Italiano de Buenos Aires

\section{Eduardo de Santibañes}

Hospital Italiano de Buenos Aires

Juan Pekolj

Hospital Italiano de Buenos Aires

\section{Oscar Mazza}

Hospital Italiano de Buenos Aires

Martín de Santibañes

Hospital Italiano de Buenos Aires

\section{Research Article}

Keywords: neutrophil-to-lymphocyte ratio, survival analysis, pancreatic cancer, perineural invasion.

Posted Date: December 14th, 2021

DOI: https://doi.org/10.21203/rs.3.rs-1157384/v1

License: (1) (1) This work is licensed under a Creative Commons Attribution 4.0 International License. Read Full License 


\section{Abstract}

Background: The aim of this study is to analyze the role of neutrophil-lymphocyte ratio and its variation pre and post-operatively (delta NLR) in overall survival after pancreatectomy for pancreatic ductal adenocarcinoma at a single centre and to identify factors associated with overall survival.

Methods: A retrospective study of consecutive patients undergoing pancreatic resection due to PDAC or undifferentiated carcinoma from January 2010 to January 2020 was performed. Association between evaluated factors and overall survival were analyzed using a log-rank test and Cox proportional hazard regression model.

Results: Overall, 242 patients underwent complete pancreatic resection for PDAC or undifferentiated carcinoma. OS was 22.8 months ( $95 \% \mathrm{Cl}$ : 19.5-29) and survival rates at 1, 3 and 5 years were $72 \%, 32.5 \%$ and $20.8 \%$ respectively. NLR and delta NLR were not significantly associated with survival $(H R=1.14$, $95 \% \mathrm{Cl}: 0.77-1.68, \mathrm{p}=0.5)$. Lymph node ratio was significantly associated $(\mathrm{HR}=1.66,95 \% \mathrm{Cl}: 1.21-2.26$, $\mathrm{p}=0.001)$ in the bivariate analysis. In multivariable analysis the only factors that were significantly associated with survival were perineural invasion ( $\mathrm{HR}=1.94,95 \% \mathrm{Cl}: 1.21-3.14, \mathrm{p}=0.006)$, surgical margin $(\mathrm{HR}=1.83,95 \% \mathrm{Cl}: 1.10-3.02, \mathrm{p}=0.019)$, tumor size $(\mathrm{HR}=1.01,95 \% \mathrm{Cl}: 1.003-1.027, \mathrm{p}=0.16)$, postoperative CA 19-9 level $(H R=1.001, p<0.001)$, and completion of adjuvant treatment $(H R=0.53,95 \% \mathrm{Cl}: 0.35-0.8$, $\mathrm{p}=0.002)$.

Conclusion: Neutrophil-lymphocyte ratio and delta NLR were not associated with overall survival in this cohort. Risk factors such as perineural invasion, surgical margins, CA19-9 level and tumor size showed worse survival in this study, whereas completing adjuvant treatment was a protective factor.

\section{Background}

Pancreatic ductal adenocarcinoma (PDAC) is an aggressive disease with 458918 new cases diagnosed each year worldwide [1]. It is the fourth leading cause of cancer-related death in the United States, and it is predicted to become the second leading cause by 2030 [2]. In addition, it is expected to be the second cause of death related to cancer in the next decade in western countries, due improvements in other tumor treatments that have increased their overall survival at a faster rate than pancreatic cancer treatment. Moreover, the aging of the general population, who are more susceptible to cancer than young people, also contributes to the prediction of a rise in pancreas cancer mortality in the future. Surgery is involved in the only chance of cure of pancreatic cancer, but 5-year survival rates after surgical resection alone are low, approximately $10 \%$ according to different series [3,4]. Nevertheless, the development of adjuvant treatments with chemotherapy has increased survival in patients who underwent surgery $[5,6]$. In Latin America there is a lack of information about long term results after surgical treatment of pancreatic cancer. Therefore, reporting oncological outcomes after multidisciplinary treatment of this aggressive disease and identifying factors associated with overall survival is an important step towards understanding this disease. 
Currently, several studies have identified different prognostic oncological factors such as surgical margins, serum CA 19-9 level, vascular resection, and pathological characteristics such as perineural or perivascular invasion [7]. Firstly, perineural invasion (PNI) is defined as the presence of cancer cells along nerves and/or within the epineural, perineural and endoneural spaces of the neuronal sheath. Despite the fact that it has been described in several solid tumors, PNI has its highest prevalence in pancreatic ductal adenocarcinoma with a range varying between $70 \%$ and $98 \%$ [8]. Moreover, PNI is detected in nearly three quarters of the early stages of PDAC and in microscopic PDAC, suggesting that it could represent an early event in cancer progression $[9,10]$.The role of $\mathrm{PNI}$ in cancer research has been traditionally underestimated since it was considered as a non-active process, with less relevance compared with hematogenous and lymphatic spread. But new evidence shows neural regulation in cancer and cancercell induced axonogenesis [11].

Interestingly, changes in the systemic inflammatory response to tumor cell manifestation of systemic inflammation can be measured by blood-based parameters. In particular, the neutrophil-lymphocyte ratio (NLR) has been assumed to be an easily available and trustable marker to predict patients' survival in different types of patients with primary operable cancer, such as colorrectal, gastroesophageal, renal, hepatocarcinoma and lung cancer [12]. In PDAC, controversial data exist regarding NLR [13,14]. We consider patients and their disease or treatment as dynamic factors which constantly interact with each other rather than static elements. Therefore, it would be interesting to find a predictor that reflects that taking into account changes in inflammatory parameters before and after surgical treatment. Delta NLR, or the difference between postoperative and preoperative values can be expressed as an increasing or decreasing trend. This parameter has been proven useful in other tumor types, consequently, we included this variable with special interest in our study.

Another element that has been described as a prognostic factor in previous studies is lymph node ratio (LNR), expressed as the rate of positive lymph nodes over total lymph nodes harvested. However, the prognostic power of $\mathrm{N}$-status, number of examined LNs, number of positive lymph nodes, and LNR differed substantially across studies $[15,16]$.

The identification of prognostic factors might enable a better risk stratification for adjuvant treatment modalities after surgery, tending towards a more personalized treatment. Results regarding overall survival and disease-free survival of our cohort were reported previously in another language [17]. Furthermore, in this study we aim to analyze the role of neutrophil-lymphocyte ratio and variation of neutrophil-lymphocyte ratio in overall survival after pancreatectomy for pancreatic ductal adenocarcinoma in a cohort from a single, academic, high-volume pancreatic centre.

\section{Methods}

\section{Population}


A retrospective analysis was performed of a cohort with all the patients who received pancreatic resection due to PDAC or undifferentiated carcinoma from January 2010 to January 2020 in a single center. Patients were eligible to be included in the study if they were 18 years of age or older, had undergone a pancreatic resection surgery and the pathology report informed pancreatic adenocarcinoma or undifferentiated carcinoma. Overall survival (OS) was measured from surgery day to death and diseasefree survival (DFS), was measured from surgery day to recurrence detected by imaging testing and/or increase of tumoral marker according to findings in electronic records of medical history. In cases where data could not be found on medical records, national registries or relatives were reached to obtain missing information. This study was performed according to national and international regulations. The institutional Ethics Committee for Investigative Protocols approved the study protocol (No. 2836)

Baseline characteristics collected were age, gender, pancreatic surgery extent, venous resection involvement, preoperative and postoperative CA 19-9 levels, preoperative and postoperative lymphocytes and neutrophils count, anatomopathological diagnosis, tumor differentiation, maximum diameter of the tumor, surgical margins, lymph nodes harvested, lymph nodes compromised by tumoral cells, presence of perineural invasion, presence of perivascular infiltration, staging according 8th edition of TNM staging system, completion of adjuvant or neoadjuvant treatment [18]. Regarding surgical margins, R0 resection was defined as the absence of any cancer cells within $1 \mathrm{~mm}$ of any cut surface of the resected specimen. An R1 resection was defined as at least 1 cancer cell within $1 \mathrm{~mm}$ of any surface of the removed specimen. NLR was calculated by dividing the absolute neutrophils count by the absolute lymphocytes count. Preoperative NLR was measured in the routine preoperative work up, within one month from surgery. Postoperative NLR was measured one month after surgery, taking into account that no acute infection or complications that could have raised neutrophil count were present. Delta NLR was calculated by subtracting NLR postoperative value to preoperative NLR and two groups were defined according to increasing $(>0)$ or decreasing $(<0)$ trend. Likewise, lymph node ratio was also dichotomized in groups with $L N R>0.1$ and $<0.1$ as it was considered to be the optimal cut-off point in previous reports [18].

\section{Statistical Analysis}

Continuous variables were reported as median and range or mean and standard deviation when appropriate. Categorical variables were presented as number of frequency and percentages. The comparison between subgroups was carried out using Student's $t$ test or Mann-Whitney $U$ test for continuous variables. Categorical variables were compared by the $\mathrm{Chi}^{2}$ test or Fisher exact test when necessary. Survival probability was estimated according to the Kaplan-Meier method, whereas the logrank test was used for comparison of survival in different groups. Statistical significance was set at 0.05 . Bivariate and multivariable survival analysis were performed using the Cox proportional hazards model, with results presented as hazard ratio (HR) with a 95\% confidence interval (Cl). Variables that were significant in bivariate analysis were included in the final multivariable analysis. Patients with missing data were excluded from the analysis. Statistical analysis was performed in R version 3.6.3 (R Core Team 2020, Vienna, Austria) 


\section{Results}

Overall, 748 patients underwent consecutive pancreatic resection from January 2010 to January 2020. PDAC diagnosis was found in 230 and undifferentiated carcinoma was found in 12 pathology reports during the study interval counting a total of 242 pancreatic cancer subjects. Patient demographic and tumor characteristics are described in Table 1. As shown in a previous publication of our group, this cohort OS was 22.8 months (95\% Cl: 19.5-29) and survival at 1-year, 3-years and 5-years were $72 \%, 32.5 \%$ and $20.8 \%$ respectively. Median follow up was 17.8 months. DFS was calculated over 194 patients $(80.1 \%)$ due to missing data of this variable in 48 records. The median disease-free survival (DFS) was 13.8 months (95\% Cl: $12-17.6)$ and $1-3-5$ years probabilities were $56.1 \%, 21.8 \%$ and $19.4 \%$ respectively. 
Table 1

Demographic and Characteristics of the Patients at Baseline

\section{Characteristic}

Age

Male sex

Preoperative CA 19-9

Postoperative CA 19-9

Preoperative neutrophil lymphocyte ratio

Postoperative neutrophil

lymphocyte ratio

Surgical resection

\begin{tabular}{ll} 
Pancreatoduodenectomy & $165(68.2 \%)$ \\
\hline Distal pancreatectomy & $49(20.2 \%)$ \\
\hline Total pancreatectomy & $28(11.6 \%)$
\end{tabular}

Status of surgical margins

Ro

R1
Median (Interquartile Range) or

Frequency (percentage) $n=242$

$67 y r(60.3-73.8)$

$117(48.3 \%)$

$120.7(30-472.3)$

$21.4(6.8-98.4)$

$2.6(1.9-3.4)$

$2.8(1.5-3.6)$

Status of surgical margins

Vascular resection

$151(62.4 \%)$

$91(37.6 \%)$

Tumor histology

Ductal adenocarcinoma $230(95 \%)$

Undifferentiated

carcinoma

$31(12.8 \%)$

$12(5 \%)$

Differentiation

Well differentiation

Moderate differentiation

Poor differentiation

Tumor size (mm)

Lymphovascular invasion
$87(36 \%)$

$120(49.6 \%)$

$35(14.4 \%)$

$30(4-120)$

$163(67.3 \%)$

*Tumor stage was assessed according to the 8th edition of TNM staging system of pancreatic cancer by AJCC/UICC (2018). 


\section{Characteristic}

Perineural invasion

Lymph nodes harvested

Positive lymph nodes

Tumor stage*

\begin{tabular}{|c|c|c|}
\hline & IA & $18(7.4 \%)$ \\
\hline & IB & $65(26.9 \%)$ \\
\hline & $\| A$ & $16(6.6 \%)$ \\
\hline & IIB & $99(40.9 \%)$ \\
\hline & III & $44(18.2 \%)$ \\
\hline \multicolumn{3}{|l|}{ Adjuvant therapy } \\
\hline & Complete & $106(43.8 \%)$ \\
\hline & Incomplete & $26(10.7 \%)$ \\
\hline & No adjuvant therapy & 39 (16.1\%) \\
\hline & Missing data & $63(26 \%)$ \\
\hline \multicolumn{2}{|l|}{ Neoadjuvant therapy } & $8(3.3 \%)$ \\
\hline
\end{tabular}

Delta NLR (Fig. 1) was not significant in the bivariate analysis (HR=1.14, 95\% Cl: 0.77-1.68, $p=0.5)$ therefore it was not included in the multivariable analysis. Individually, preoperative neutrophil lymphocyte ratio ( $\mathrm{HR}=0.98,95 \% \mathrm{Cl}: 0.86-1.1, \mathrm{p}=0.69)$ and postoperative neutrophil lymphocyte ratio $(\mathrm{HR}=1,95 \% \mathrm{Cl}: 0.89-1.1, \mathrm{p}=0.9)$ were not significant either. The lymph node ratio was significantly associated ( $\mathrm{HR}=1.66,95 \% \mathrm{Cl}: 1.21-2.26, \mathrm{p}=0.001)$ in the bivariate analysis (Table 2), but it was not significant in multivariable analysis $(\mathrm{HR}=1.1,95 \% \mathrm{Cl}$ : 0.6-2.01, $\mathrm{p}=0.74)$. Perineural invasion was significantly associated with worse survival in bivariate analysis $(\mathrm{HR}=1.53,95 \% \mathrm{Cl}: 1.063-2.219, \mathrm{p}=0.02)$ (Fig. 2). Other variables that were identified as risk factors in bivariate analysis were surgical resection (total pancreatectoduodenectomy $\mathrm{HR}=2.2,95 \% \mathrm{Cl}: 1.45-3.37, \mathrm{p}<0.001)$, vascular resection $(\mathrm{HR}=1.92$, 95\%Cl: 1.27-2.9, $p=0.002)$, postoperative CA 19-9 level $(H R=1.0001,95 \% \mathrm{Cl}: 1-1.001, p<0.001)$, tumor size ( $\mathrm{HR}=1.01,95 \% \mathrm{Cl}: 1.002-1.019, \mathrm{p}=0.02)$, positive lymph nodes $(\mathrm{HR}=1.09,95 \% \mathrm{Cl}: 1.032-1.15, \mathrm{p}=0.002)$, surgical margins $(H R=1.63,95 \% \mathrm{Cl}: 1.186-2.248, p=0.003)$, TNM stage III $(H R=2.31,95 \% C l: 1.14-4.69$, $\mathrm{p}=0.02$ ). Finally, having completed adjuvant treatment with chemotherapy was a protective factor since it was associated with better survival $(H R=0.43,95 \% \mathrm{Cl}$ : 0.30-0.62, $p<0.001)$. 
Page 8/17 
Table 2

Bivariate and multivariate analysis results.

\begin{tabular}{|c|c|c|c|c|c|c|}
\hline \multirow[t]{3}{*}{ Factors } & \multicolumn{3}{|c|}{ Bivariate } & \multicolumn{3}{|c|}{ Multivariate } \\
\hline & HR & IC 95\% & P-value & HR & IC 95\% & P-value \\
\hline & & & & (adjusted) & & \\
\hline Age & 1.004 & $0.99-1.02$ & 0.6 & & & \\
\hline Sex & 1.224 & $0.90-1.67$ & 0.2 & & & \\
\hline Preoperative NLR & 0.98 & $0.86-1.1$ & 0.69 & & & \\
\hline Postoperative NLR & 1 & $0.89-1.1$ & 0.9 & & & \\
\hline Delta NLR & 1.14 & $0.77-1.68$ & 0.5 & & & \\
\hline Surgical resection & 1.095 & $1.03-1.16$ & 0.005 & 1.06 & $0.45-2.45$ & 0.89 \\
\hline Vascular resection & 1.915 & $1.26-2.9$ & 0.002 & 0.83 & $0.41-1.69$ & 0.61 \\
\hline Preoperative CA 19-9 & 1 & 1 & 0.08 & & & \\
\hline Postoperative CA 19-9 & 1.001 & 1 & 1.001 & 1.0006 & $\begin{array}{l}1.0003- \\
1.0009\end{array}$ & 0.00039 \\
\hline Tumor histology & 0.54 & $0.22-1.33$ & 0.2 & & & \\
\hline Differentiation & 1.12 & $0.9-1.4$ & 0.3 & & & \\
\hline Tumor size & 1.01 & $\begin{array}{l}1.002- \\
1.019\end{array}$ & 0.02 & 0.015 & $\begin{array}{l}1.0027- \\
1.027\end{array}$ & 0.016 \\
\hline Lymph nodes harvested & 1.01 & $0.98-1.03$ & 0.9 & & & \\
\hline Positive lymph nodes & 1.09 & $1.03-1.15$ & 0.002 & 0.96 & $0.81-1.14$ & 0.68 \\
\hline Lymph node ratio & 1.66 & $1.21-2.26$ & 0.001 & 1.11 & $0.61-2.01$ & 0.74 \\
\hline $\mathrm{R} 1$ resection & 1.63 & $1.19-2.25$ & 0.003 & 1.83 & $1.1-3.03$ & 0.019 \\
\hline Perineural invasion & 1.54 & $1.06-2.22$ & 0.02 & 1.94 & $1.21-3.14$ & 0.006 \\
\hline Perivascular invasion & 1.263 & $0.89-1.8$ & 0.2 & & & \\
\hline IA stage & 0.6 & $0.32-1.15$ & 0.1 & & & \\
\hline IB stage & 0.8 & $0.56-1.16$ & 0.2 & & & \\
\hline IIA stage & 0.56 & $0.27-1.14$ & 1 & & & \\
\hline IIB stage & 1.22 & $0.89-1.67$ & 0.2 & & & \\
\hline III stage & 1.55 & $1.07-2.26$ & 0.02 & 1.57 & $0.62-3.97$ & 0.34 \\
\hline
\end{tabular}




\begin{tabular}{|lllllll|}
\hline Factors & \multicolumn{2}{l}{ Bivariate } & \multicolumn{4}{l|}{ Multivariate } \\
\hline $\begin{array}{l}\text { Complete adjuvant } \\
\text { chemotherapy }\end{array}$ & 0.43 & $0.31-0.62$ & $<0.0001$ & 0.53 & $0.35-0.798$ & 0.002 \\
\hline NLR= neutrophil-lymphocyte ratio & & & & & \\
\hline
\end{tabular}

\section{Multivariable Analysis}

In the multivariable analysis including the variables which resulted statistically significant in bivariate analysis, the risk factors that remained significantly associated with worse overall survival were the presence of perineural invasion ( $\mathrm{HR}=1.94,95 \% \mathrm{Cl}$ : $1.21-3.14, \mathrm{p}=0.006)$, surgical margin $(\mathrm{HR}=1.83,95 \% \mathrm{Cl}$ : 1.10-3.02, $p=0.019)$, tumor size ( $\mathrm{HR}=1.01,95 \% \mathrm{Cl}: 1.003-1.027, \mathrm{p}=0.16)$ and postoperative CA 19-9 level $(H R=1.001,95 \%$ IC: $1.0003-1.0009, p<0.001)$. On the other hand, the protective factor of completion of adjuvant treatment $(\mathrm{HR}=0.53,95 \% \mathrm{Cl}: 0.35-0.8, \mathrm{p}=0.002)$ remained significant. The final model with 110 subjects (132 observations deleted due to missingness of any of the variables) had a c-statistic of 0.69.

\section{Adjuvant Treatment}

The most used adjuvant treatment was gemcitabine based therapies in 86 patients (35.6\%), whereas monotherapy or in association with oxaliplatin or capecitabine. FOLFIRINOX was used in 13 patients (5.3\%). Radiotherapy was used in 33 patients (13.6\%). The use of neoadjuvant treatment did not decrease the frequency of $\mathrm{PNI}$ in our cohort $(\mathrm{OR}=1.2,95 \% \mathrm{Cl}$ : $0.18-6.5)$ but due to a low number of subjects (8 patients) in the neoadjuvant group this result should be taken with caution.

\section{Discussion}

Surgical treatment is the only potentially curative treatment for patients with pancreatic cancer that present at a resectable stage and it may be substantially underutilized even in countries with sufficient medical resources [20]. Hence the importance of reporting long term results of surgical treatment of this disease. In addition, little evidence has been published from Argentina and from Latin America about long term oncological outcomes of multidisciplinary treatment of pancreatic cancer. When we analyze the overall survival in this cohort of 10 years of consecutive pancreatectomies for PDAC in a single center, we find that results are similar to international standards. For example, in a recent manuscript from Heidelberg University, 937 patients were included that received pancreatic surgery over the first decade of this millennium and they obtained a median actual overall survival of 22.1 months and 5-year survival rate of $17 \%$ [7]. Whereas in this cohort estimated overall survival was 22.8 months and 5 -year survival rate was $20.8 \%$.

Perineural invasion is a major pathway by which tumors progress and spread to the adjacent tissue. Furthermore, it has been shown to be an important prognostic factor in many types of human malignancies [21]. The pancreas is an organ that is highly innervated by sympathetic and 
parasympathetic nerves from ganglia around the celiac and superior mesenteric artery [22]. As mentioned before, recent studies have proposed a major role of PNI in cancer progression, to the point that in animal models of PDAC the ablation of sympathetic nerves resulted in the inhibition of cancer progression [23]. The aggressiveness of PNI cancer cell invasion has been related to neuropathic changes, desmoplasia, and pain. Severe and enduring pain has been strongly associated with poor prognosis in PDAC [8, 24]. In our study we have found using multivariable analysis, that presence of PNI was an independent prognostic factor for worse OS, with an HR of 1.94. These results are consistent with findings reported by a recent multicenter study which included 778 patients from different world wide known pancreas centers. They found that PNI was an independent predictor of survival with a HR of 1.6, and median OS was 50 months in patients without PNI compared with 27 months in those with PNI. We concur with the authors that perineural invasion plays a major role in disease recurrence and survival after pancreatectomy for pancreatic head ductal adenocarcinoma, especially in early stage disease [25].

The presence or absence of lymph node metastases is an established prognostic factor in patients with resected adenocarcinoma of the pancreas. In a reported experience from Johns Hopkins University of 905 patients that underwent pancreaticoduodenectomy for pancreatic adenocarcinoma from 1995 to 2005, they obtained a median survival for all patients of 17.4 months, and the 5 -year actuarial survival rate was $16.1 \%$. Demographics, operative data, number of lymph nodes evaluated, number of lymph nodes with metastatic carcinoma, LN ratio, pathologic margin status, and long-term survival were analysed. In that cohort it was found that LN ratio was one of the most powerful predictors of survival [26]. In our study LN ratio was also a significant finding in bivariate analysis but was not significant after adjusting for the other factors.

Systemic inflammation is a well-established physiopathological factor in neoplastic condition. Neutrophilia reflects an underlying neoplastic inflammatory syndrome and is usually accompanied by a variable degree of lymphopenia. Therefore, neutrophil lymphocyte ratio (NLR) is an accessible marker of systemic inflammation. The balance between the negative effects of neutrophilia and the positive effects of lymphocyte-mediated immunity is an interesting line of research. There are meta-analyses that have validated pretreatment NLR as a prognostic factor, and high NLR values have been linked to poor outcomes in numerous types of solid tumors [27]. In metastatic pancreatic adenocarcinoma, a high neutrophil lymphocyte ratio at diagnosis is a marker of poor prognosis. In addition, a high preoperative NLR indicates a worse prognosis than in patients with a low NLR according to another meta-analysis [28]. Furthermore, the prognostic role of baseline NLR and NLR variation after surgery is a novel approach that, to our knowledge, has not been studied before. Therefore, we included this variable in the analysis with special interest, but an association with survival was not found in this cohort. Nevertheless, prospective studies would be appropriate to validate this finding due to the possibility of information bias related to the retrospective nature of this work.

Different authors have found a significantly lower rate of PNI in patients receiving preoperative chemotherapy/chemoradiation (50-70\%) compared with those undergoing immediate resection (80-90\%) $[29,30]$. In our cohort only 8 patients $(3.3 \%)$ of the surgically resected patients received neoadjuvant 
therapy. We could not find a statistically significant difference between groups but, due to the low number of patients in the neoadjuvant arm, it is not proper to draw conclusions about the effect of neoadjuvant treatment on PNI. Whereby in our institution neoadjuvant therapy approach is reserved for borderline and locally advanced tumors, previously discussed in a multidisciplinary tumor board. Interestingly, recent evidence on this topic has been revealed in the PREOPANC Trial, which was a randomized phase III trial in 16 centers from Netherlands where they compared patients with resectable or borderline resectable pancreatic cancer that were randomly assigned to receive preoperative chemoradiotherapy versus immediate surgery and 6 courses of adjuvant gemcitabine. The patients that underwent upfront surgery showed a median overall survival of 16 months vs 14.3 months for the neoadjuvant arm $(P=0.096)$. Even though they didn't reach statistical significance in the primary endpoint that was overall survival, a significantly higher proportion of patients reached R0 resections in the neoadjuvant arm ( $71 \%$ vs $40 \%$, $\mathrm{P}<0.001)$ and also a lower rate of perineural invasion [31]. In a posterior long-term analysis from this trial, three- and five-year overall survival (intention-to-treat) was $27.7 \%$ and $20.5 \%$ after preoperative CRT versus $16.5 \%$ and $6.5 \%$ after immediate surgery (HR $0.73 ; 95 \% \mathrm{Cl} 0.56$ to $0.96 ; \mathrm{p}=0.025)$, which becomes an important evidence towards neoadjuvant approach in this group [32].

This study is not free from limitations. Its retrospective nature makes it susceptible to information bias. Some of the follow up data is also a problem with these patients, especially in our center. Being a referral center for pancreatic diseases for patients all over Argentina, many patients receive surgery in our institution but afterwards they must continue their follow-up in their home towns or cities, accounting for loss of some oncological outcomes such as recurrence. On the other hand, information about death could be recovered by different means, such as telephonic interviews and public registries making practically a complete registry of this outcome. Furthermore, to the best of our knowledge, there is no previous evidence of the study of variation of neutrophil-lymphocyte ratio in pancreatic cancer surgery survival. Finally, the main use of these results for our daily practice is related to the awareness of physicians and patients about the prognostic factors that may lead to long term survival after surgery.

\section{Conclusion}

Neutrophil-lymphocyte ratio and delta NLR were not associated with OS in a ten years cohort of patients with PDAC surgically treated. The presence of perineural invasion was clearly associated with worse OS. Other risk factors such as surgical margins, CA19-9 level and tumor size also showed worse survival in our cohort, whereas completing adjuvant treatment was a protective factor.

\section{Declarations}

\section{AUTHOR CONTRIBUTIONS}

(I) Conception and design: Martín de Santibañes, Ignacio G. Merlo

(II) Administrative support: Juan Pekolj

(III) Provision of study materials or patients: Oscar Mazza, Rodrigo Sanchez Claria, Juan Pekolj, Martín 
de Santibañes, Eduardo de Santibañes

(IV) Collection and assembly of data: Ignacio G. Merlo, Eugenia Fratantoni

(V) Data analysis and interpretation: Ignacio G. Merlo, Victoria Ardiles

(VI) Manuscript writing: All authors

(VII) Final approval of manuscript: All authors

The authors are accountable for all aspects of the work in ensuring that questions related to the accuracy or integrity of any part of the work are appropriately investigated and resolved.

\section{References}

1. F. Bray, J. Ferlay, I. Soerjomataram, R. L. Siegel, L. A. Torre, and A. Jemal, “Global cancer statistics 2018: GLOBOCAN estimates of incidence and mortality worldwide for 36 cancers in 185 countries," CA. Cancer J. Clin., vol. 68, no. 6, pp. 394-424, 2018. PMID: 30207593

2. L. Rahib, B. D. Smith, R. Aizenberg, A. B. Rosenzweig, J. M. Fleshman, and L. M. Matrisian, "Projecting cancer incidence and deaths to 2030: The unexpected burden of thyroid, liver, and pancreas cancers in the united states," Cancer Res., vol. 74, no. 11, pp. 2913-2921, 2014. PMID: 24840647

3. H. Oettle, P. Neuhaus, A. Hochhaus, J. T. Hartmann, K. Gellert, K. Ridwelski, et al., "Adjuvant chemotherapy with gemcitabine and long-term outcomes among patients with resected pancreatic cancer: The CONKO-001 randomized trial," JAMA - J. Am. Med. Assoc., vol. 310, no. 14, pp. 14731481, 2013. PMID: 24104372

4. J. P. Neoptolemos, J. Kleeff, P. Michl, E. Costello, W. Greenhalf, and D. H. Palmer, "Therapeutic developments in pancreatic cancer: Current and future perspectives," Nat. Rev. Gastroenterol. Hepatol., vol. 15, no. 6, pp. 333-348, 2018. PMID: 29717230

5. U. Klaiber, T. Hackert, and J. P. Neoptolemos, "Adjuvant treatment for pancreatic cancer," Transl. Gastroenterol. Hepatol., vol. 4, no. April, 2019. PMID: 31143848

6. T. Conroy, P. Hammel, M. Hebbar, M. Ben Abdelghani, A.C. Wei, J.-L. Raoul, et al., "FOLFIRINOX or Gemcitabine as Adjuvant Therapy for Pancreatic Cancer," N Engl J Med 2018;379:2395-406. PMID: 30575490

7. O. Strobel, P. Lorenz, U. Hinz, M. Gaida, A.K. Konig, T. Hank, et al., "Actual Five-year Survival After Upfront Resection for Pancreatic Ductal Adenocarcinoma," Ann. Surg., Jul. 2020. PMID: 32649469 doi: 10.1097/SLA.0000000000004147

8. F. Liebl, I. E. Demir, K. Mayer, T. Schuster, J. D'Haese, K. Becker, et al., "The impact of neural invasion severity in gastrointestinal malignancies: A Clinicopathological Study," Ann. Surg., vol. 260, no. 5 , pp. 900-908, 2014. PMID: 25379860

9. C. Luchini, N. Veronese, A. Nottegar, G. Riva, C. Pilati, A. Mafficini, et al., "Perineural Invasion is a Strong Prognostic Moderator in Ampulla of Vater Carcinoma: A Meta-analysis," Pancreas, vol. 48, no. 1, pp. 70-76, 2019. PMID: 30451797 
10. G. Gasparini, M. Pellegatta, S. Crippa, M. Schiavo Lena, G. Belfiori, et al., "Nerves and pancreatic cancer: New insights into a dangerous relationship," Cancers (Basel)., vol. 11, no. 7, pp. 1-24, 2019. PMID: 31248001

11. S. Faulkner, P. Jobling, B. March, C. C. Jiang, and H. Hondermarck, "Tumor neurobiology and the war of nerves in cancer," Cancer Discov., vol. 9, no. 6, pp. 702-710, 2019. PMID: 30944117

12. C. S. D. Roxburgh and D. C. McMillan, "Role of systemic inflammatory response in predicting survival in patients with primary operable cancer," Futur. Oncol., vol. 6, no. 1, pp. 149-163, 2010. PMID: 20021215

13. G. Luo, M. Guo, Z. Liu, Z. Xiao, K. Jin, J. Long, et al., "Blood Neutrophil-Lymphocyte Ratio Predicts Survival in Patients with Advanced Pancreatic Cancer Treated with Chemotherapy," Ann. Surg. Oncol., vol. 22, no. 2, pp. 670-676, 2015. PMID: 25155401

14. Y. Zhou, Q. Wei, J. Fan, S. Cheng, W. Ding, and Z. Hua, "Prognostic role of the neutrophil-tolymphocyte ratio in pancreatic cancer: A meta-analysis containing 8252 patients," Clin. Chim. Acta, vol. 479, no. January, pp. 181-189, 2018. PMID: 29407690

15. G. Malleo, L. Maggino, P. Capelli, F. Gulino, S. Segattini, A. Scarpa, et al., "Reappraisal of Nodal Staging and Study of Lymph Node Station Involvement in Pancreaticoduodenectomy with the Standard International Study Group of Pancreatic Surgery Definition of Lymphadenectomy for Cancer," J. Am. Coll. Surg., vol. 221, no. 2, pp. 367-379.e4, 2015. PMID: 26081176

16. M. S. You, S. H. Lee, Y. H. Cho, B. S. Shin, W. H. Paik, J. K. Riu, et al., "Lymph node ratio as valuable predictor in pancreatic cancer treated with RO resection and adjuvant treatment," BMC Cancer, vol. 19, no. 1, pp. 1-8, 2019. PMID: 31615457

17. I. Merlo, E. Fratantoni, M. de Santibañes, V. Ardiles, R. Sanchez Clariá, J. Pekolj, E. de Santibañes, O. Mazza “Long-term survival after pancreatic cancer surgery” Medicina (B Aires). 2021;81(5):800-807. PMID: 34633955

18. L. Cong, Q. Liu, R. Zhang, M. Cui, X. Zhang, X. Gao, et al. "Tumor size classification of the 8th edition of TNM staging system is superior to that of the 7th edition in predicting the survival outcome of pancreatic cancer patients after radical resection and adjuvant chemotherapy," Sci Rep. 2018;8(1):10383. PMID: 29991730

19. T. Aoyama, N. Yamamoto, M. Kamiya, M. Murakawa, H. Tamagawa, S. Sawazaki, et al., "The lymph node ratio is an independent prognostic factor in pancreatic cancer patients who receive curative resection followed by adjuvant chemotherapy," Anticancer Res., vol. 38, no. 8, pp. 4877-4882, 2018. PMID: 30061263

20. D. S. Swords, S. J. Mulvihill, D. E. Skarda, S. Finlayson, G. J. Stoddard, M. J. Ott, et al., "Hospital-level Variation in Utilization of Surgery for Clinical Stage I-Il Pancreatic Adenocarcinoma," Ann. Surg., vol. 269, no. 1, pp. 133-142, 2019. PMID: 28700442

21. D. Chatterjee, M. H. Katz, A. Rashid, H. Wang, A. C. luga, G. R. Varadhachary, et al., “Perineural and Intraneural Invasion in Posttherapy Pancreaticoduodenectomy Specimens Predicts Poor Prognosis 
in Patients With Pancreatic Ductal Adenocarcinoma," Am. J. Surg. Pathol., vol. 36, no. 3, pp. 409417, Mar. 2012. PMID: 22301497

22. S. Q. Yi, K. Miwa, T. Ohta, M. Kayahara, H. Kitagawa, A. Tanaka, et al., "Innervation of the pancreas from the perspective of perineural invasion of pancreatic cancer," Pancreas, vol. 27, no. 3, pp. 225229, 2003. PMID: 14508126

23. B. W. Renz, R. Takahashi, T. Tanaka, M. Macchini, Y. Hayakawa, Z. Dantes, et al., " $\beta 2$ AdrenergicNeurotrophin Feedforward Loop Promotes Pancreatic Cancer," Cancer Cell, vol. 33, no. 1, pp. 7590.e7, 2018. PMID: 29249692

24. G. O. Ceyhan, F. Bergmann, M. Kadihasanoglu, B. Altintas, I. E. Demir, U. Hinz, et al., "Pancreatic Neuropathy and Neuropathic Pain-A Comprehensive Pathomorphological Study of 546 Cases," Gastroenterology, vol. 136, no. 1, pp. 177-186.e1, 2009. PMID: 18992743

25. S. Crippa, I. Pergolini, A. A. Javed, K. C. Honselmann, M. J. Weiss, F. Di Salvo, et al., "Implications of Perineural Invasion on Disease Recurrence and Survival After Pancreatectomy for Pancreatic Head Ductal Adenocarcinoma," Ann. Surg., vol. Publish Ah, Oct. 2020. PMID: 33086324

26. T. M. Pawlik, A. L. Gleisner, J. L. Cameron, J. M. Winter, L. Assumpcao, K. D. Lillemoe, et al., "Prognostic relevance of lymph node ratio following pancreaticoduodenectomy for pancreatic cancer," Surgery, vol. 141, no. 5, pp. 610-618, 2007. PMID: 17462460

27. N. Choi, J. H. Kim, E. K. Chie, J. Gim, and H. C. Kang, "A meta-analysis of the impact of neutrophil-tolymphocyte ratio on treatment outcomes after radiotherapy for solid tumors," Med. (United States), vol. 98 , no. 18, pp. 1-8, 2019. PMID: 31045780

28. P. McLellan, J. Henriques, F. Ksontini, S. Doat, P. Hammel, J. Desrame, et al., "Prognostic value of the early change in neutrophil-to-lymphocyte ratio in metastatic pancreatic adenocarcinoma," Clin. Res. Hepatol. Gastroenterol, 45(3):101541, 2021. PMID: 33055007

29. C. R. Ferrone, G. Marchegiani, T. S. Hong, D. P. Ryan, V. Deshpande, E. I. McDonnell, et al., "Radiological and surgical implications of neoadjuvant treatment with FOLFIRINOX for locally advanced and borderline resectable pancreatic cancer," Ann. Surg., vol. 261, no. 1, pp. 12-17, 2015. PMID: 25599322

30. C. A. Barnes, M. I. Chavez, S. Tsai, M. Aldakkak, B. George, P. S. Ritch, et al., "Survival of patients with borderline resectable pancreatic cancer who received neoadjuvant therapy and surgery," Surg. (United States), vol. 166, no. 3, pp. 277-285, 2019. PMID: 31272811

31. E. Versteijne, M. Suker, K. Groothuis, J. M. Akkermans-Vogelaar, M. G. Besselink, B. A. Bonsing, et al., "Preoperative Chemoradiotherapy Versus Immediate Surgery for Resectable and Borderline Resectable Pancreatic Cancer: Results of the Dutch Randomized Phase III PREOPANC Trial.," J. Clin. Oncol., vol. 38, no. 16, pp. 1763-1773, Jun. 2020. PMID: 32105518

32. C. H. J. Van Eijck et al., "Preoperative chemoradiotherapy to improve overall survival in pancreatic cancer: Long-term results of the multicenter randomized phase III PREOPANC trial.," J. Clin. Oncol., vol. 39, no. 15_suppl, pp. 4016-4016, May 2021. doi: 10.1200/JC0.2021.39.15_suppl.4016 
Figures

\section{Kaplan-Meier}

Strata + Negative Delta NLR + Positive Delta NLR

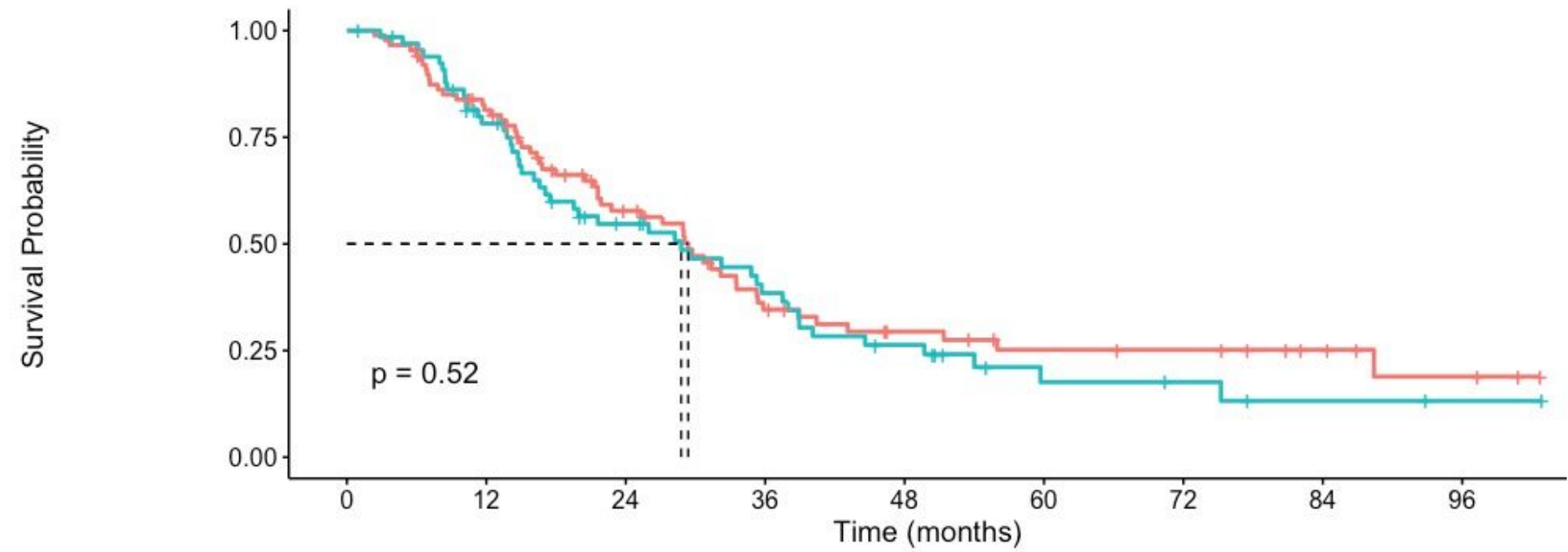

Number at risk

\begin{tabular}{|c|c|c|c|c|c|c|c|c|c|}
\hline 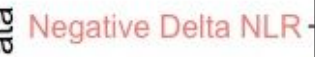 & 88 & 67 & 40 & 22 & 15 & 11 & 10 & 6 & 3 \\
\hline Fositive Delta NLR & 67 & 48 & 29 & 19 & 12 & 5 & 4 & 2 & 1 \\
\hline & 0 & 12 & 24 & 36 & 48 & 60 & 72 & 84 & 96 \\
\hline
\end{tabular}

\section{Figure 1}

Kaplan-Meier curves according to variation of postoperative and preoperative neutrophil lymphocyte ratio (delta NLR). 
Kaplan-Meier of Perineural Invasion

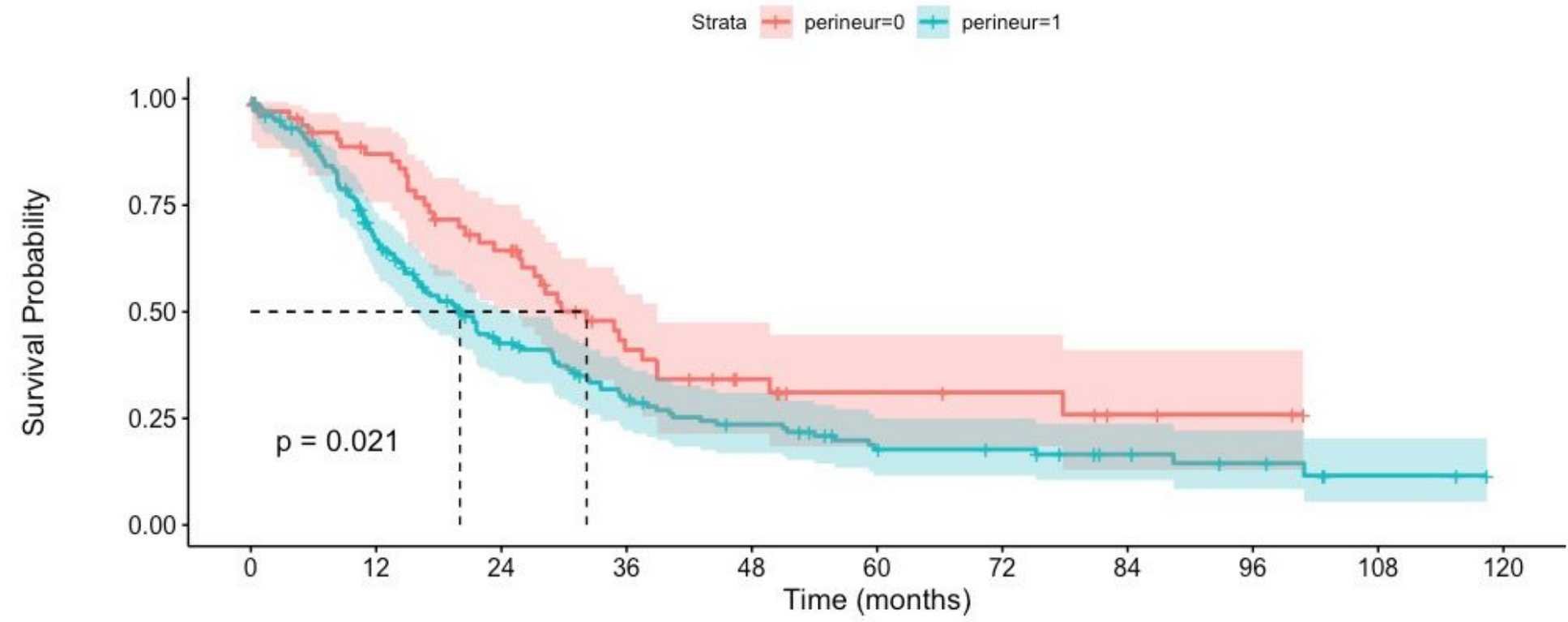

Number at risk

\begin{tabular}{|c|c|c|c|c|c|c|c|c|c|c|c|}
\hline \multirow{3}{*}{ 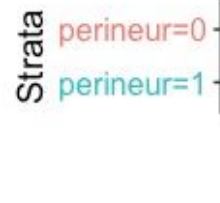 } & 67 & 51 & 35 & 18 & 11 & 7 & 6 & 3 & 2 & 0 & 0 \\
\hline & 175 & 107 & 58 & 37 & 27 & 17 & 15 & 9 & 6 & 2 & 0 \\
\hline & 0 & 12 & 24 & 36 & 48 & $\begin{array}{l}60 \\
(\mathrm{mc}\end{array}$ & 72 & 84 & 96 & 108 & 120 \\
\hline
\end{tabular}

Figure 2

Kaplan-Meier curves according to the presence of perineural invasion. 